\title{
Individual differences: Either relational learning or item-specific learning in a same/different task
}

\author{
L. Caitlin Elmore, Anthony A. Wright, and Jacquelyne J. Rivera \\ University of Texas Health Science Center at Houston, Houston, Texas \\ AND \\ JEFFREY S. KATZ \\ Auburn University, Auburn, Alabama
}

\begin{abstract}
Three pigeons were trained in a three-item simultaneous same/different task. Three of six stimulus combinations were not trained (untrained set) and were tested later. Following acquisition, the subjects were tested with novel stimuli, the untrained set, training-stimulus inversions, and object shape and color manipulations. There was no novel-stimulus transfer - that is, no abstract-concept learning. Two pigeons showed partial transfer to untrained pairs and good transfer to stimulus inversions, suggesting that they had learned the relationship between the stimuli. Lack of transfer by the third pigeon suggests item-specific learning. The somewhat surprising finding of relational learning by 2 pigeons with only six training pairs suggests restricted-domain relational learning that was controlled more by color than by shape features. Individual differences of item-specific learning by 1 pigeon and relational learning by 2 others demonstrate that this task can be learned in different ways and that relational learning can occur in the absence of novel-stimulus transfer.
\end{abstract}

Learning to judge the relationships among stimuli is considered higher order learning because it requires judgments that transcend the actual features of the stimuli. $\mathrm{Ab}$ stract concepts of same and different require just such an understanding. There is no question that humans readily develop the ability to judge relationships among stimuli, and concepts like sameness have been considered to be "the very keel and backbone of our thinking" (James, 1890/1950, p. 459).

In addition to the interest in the human ability to learn abstract concepts, there is also the comparative issue of abstract-concept learning in nonhuman animals. Since the seminal publication by Zentall and Hogan in 1974 and those by David Premack beginning in 1978, there has been considerable interest in the issues of whether animals can learn abstract concepts and, if so, which species have this ability (e.g., Premack, 1978, 1983a, 1983b; Premack \& Premack, 1983). Premack (1978) argued that species without language training would not be able to learn the same/ different (S/D) concept. However, in the past 30 years, researchers have shown that many species - such as baboons, rhesus monkeys, capuchin monkeys, parrots, and even pigeons - can learn S/D abstract concepts (e.g., Bhatt \& Wright, 1992; Bovet \& Vauclair, 2001; Cook, Cavoto, \& Cavoto, 1995; Katz \& Wright, 2006; Katz, Wright, \& Bachevalier, 2002; Pepperberg, 1987; Wasserman, Hugart, \& Kirkpatrick-Steger, 1995; Wright, Rivera, Katz, \& Bachevalier, 2003; Wright, Santiago, \& Sands, 1984; Wright, Shyan, \& Jitsumori, 1990).
Simply learning an S/D task is not sufficient to demonstrate abstract-concept learning. Work in our laboratories has demonstrated that pigeons and monkeys trained in an S/D task with a small set of training items (e.g., eight items) do not demonstrate abstract-concept learning, in that they do not transfer their performance to novel items (e.g., Katz \& Wright, 2006; Wright \& Katz, 2006). This raises the following question: If an animal acquires an S/D task but does not show abstract-concept learning, how did the animal solve the task?

Various theories have been proposed to explain how animals learn S/D tasks (and match-to-sample [MTS] tasks) in the absence of abstract-concept learning. Perhaps the most notable is the suggestion by Carter and Werner (1978) that animals frequently learn sets of if-then associations, as opposed to the single abstract matching rule in MTS. In the case of an S/D task, an if-then association might read out as "if Stimulus A plus Stimulus B, then respond different" or "if Stimulus C plus Stimulus C, then respond same." Also described by Carter and Werner is the notion that subjects may be learning a configural association. According to a configural association, subjects learn to treat the sample and the probe in an S/D task as a gestalt configuration: They learn the pair of stimuli as a unique whole. Carter and Werner referred to the experiments of Lashley (1930), who trained rats in a conditioned discrimination task using upright and inverted triangles as discriminative stimuli. Both triangles appeared on a background. If the background was black, the rats were

L. C. Elmore, lauren.c.elmore@uth.tmc.edu 
to jump to the upright triangle, but if the background was striped, the rats were to jump to the inverted triangle. One way that the rats could have learned the discrimination is by gestalt-configural learning, meaning that the appropriate response to each stimulus configuration had been conditioned. They memorized what each compound signaled (e.g., an inverted triangle on a black background), instead of learning to choose triangle orientation conditional upon the background type.

In other tasks (e.g., MTS tasks), if-then associative learning and configural learning can be distinguished (Wright, 1997). This distinction is more difficult to make in S/D tasks and will not be made in the experiments in this article.

A third possibility is that pigeons could learn the relationship between the upper stimulus and the lower stimulus and associate the proper response on the basis of this relationship, but only within a limited context. Basically, the animal applies this relational rule within the domain of stimuli circumscribed by the training stimuli. We will term this condition a restricted-domain relational strategy. One can imagine that, following training with an expanding set size of stimuli in the S/D task (as in Katz \& Wright, 2006), the domain would broaden until eventually full abstract-concept learning is achieved. Additionally, it should be noted that relational learning by animals with a small set of training stimuli is not an unreasonable expectation, since infant chimpanzees trained with only two stimuli (a lock and a cup) achieved full abstract-concept learning in the context of an MTS task (Oden, Thompson, \& Premack, 1988).

The purpose of the present study was to train pigeons in a three-item S/D task, rather than an eight-item task, to increase the chances that they would learn the task itemspecifically. A series of tests were conducted to identify whether the learning was in fact item specific or relational. Stimulus manipulations (shape and color changes) were tested to explore the range over which item-specific or relational learning might be maintained.

\section{EXPERIMENT 1}

\section{Method}

\section{Subjects}

Three experimentally naive White Carneaux pigeons (Columba livia) were the subjects in Experiment 1. At the start of the experiment, all 3 pigeons were 7 years old. The pigeons were tested 5 days per week. They were maintained at approximately $85 \%$ of their freefeeding weights and were allowed free access to grit and water in their home cages. A 14:10-h light:dark cycle was maintained in the pigeons' colony room.

\section{Apparatus}

Chambers. The pigeons were trained and tested in one of two identical $(35.9 \mathrm{~cm}$ wide $\times 45.7 \mathrm{~cm}$ deep $\times 51.4 \mathrm{~cm}$ high), custommade wooden test chambers. A fan (Dayton 5C115A, Niles, IL) was installed at the back wall of each chamber to provide ventilation and white noise. Reinforcement was provided by a custom-made food hopper, which contained mixed grain. The hopper was accessed through an opening $(5.1 \times 5.7 \mathrm{~cm})$ in the center of the front panel. The opening was $3.8 \mathrm{~cm}$ above the floor of the chamber. The pigeons pecked a computer monitor, and their pecks were detected by an infrared touchscreen (17-in. Unitouch, Carroll Touch, Round Rock,
TX), which was pressure fitted within a $40.6 \times 32.1 \mathrm{~cm}$ cutout in the front panel, centered $7.7 \mathrm{~cm}$ from the top of the chamber. The 28 -V houselight (No. 1829, Chicago Miniature, Hackensack, NJ) was placed in the center of the chamber ceiling and was illuminated during the intertrial interval.

Stimuli. Digitized color pictures were presented on a $40.3-\mathrm{cm}$ color monitor (Eizo T550, Ishikawa, Japan; $800 \times 600$ pixel resolution). Each stimulus display contained two color pictures (each $5.7 \times$ $3.8 \mathrm{~cm})$ and a white rectangle $(2.5 \times 2.4 \mathrm{~cm})$ on a black background. The pictures were centered $20.63 \mathrm{~cm}$ from the left edge of the monitor screen, and the top picture was $18.75 \mathrm{~cm}$ from the top of the cutout in the front panel. The pictures were vertically aligned, with a $1.28-\mathrm{cm}$ space between them. The bottom of the white rectangle was horizontally aligned with the bottom of the lower picture. The white rectangle was placed $1.4 \mathrm{~cm}$ to the right of the lower picture. Same and different trials were composed by selecting from a set of three images: apples, a cat, and a flower. Six possible different trials could be composed from a three-item set, but only three of these six possible trials were used during initial training. These three different trials were carefully selected such that each stimulus appeared once as the sample and once as the probe, so that responses could not be learned on the basis of the sample alone. All three possible same trials were used for a total of six training displays. The six training displays are shown in Figure 1A. The display size was $68.7^{\circ}$ (vertical) $\times 72.9^{\circ}$ (horizontal), as viewed from the pigeon's perspective of $6.5 \mathrm{~cm}$ (the average beak length plus the 3 -cm distance from the screen).

Experimental control. Experimental sessions were created, controlled, and recorded using custom software written in Visual Basic on a Pentium-based personal computer. The monitor was controlled by a video card (ATI 3D Rage Pro AGP 2X, ON, Canada). A computer-relay interface (Model PI0-12, Metrabyte, Taunton, MA) was used to operate the food hopper and the houselight.

\section{Procedure}

Preliminary training. Pigeons were first trained to eat out of the hopper. Next, responses were autoshaped to a white rectangle (the same size and position as the future different response area) and to another white rectangle (the same size and position as the future same response area). Each rectangle was presented on 48 trials in a 96-trial session. The pigeons were initially taught to associate the white rectangles with the presentation of food in the hopper. This association was accomplished by presenting one of the rectangles for $10 \mathrm{sec}$, followed by the presentation of food for $4-6 \mathrm{sec}$ (depending on each pigeon's metabolism and weight). If the pigeon pecked the white rectangle during the 10 -sec interval, the rectangle was removed prior to the end of the 10 -sec interval, and food was presented for the allotted time. After one or two sessions with this procedure (depending on the individual pigeon's willingness to peck), the shaping program was changed such that the pigeons were required to peck the white rectangle in order to receive access to the food in the hopper. Once the pigeons were dependably responding to the rectangles (another 1-2 sessions), S/D training began.

S/D training. Each trial of the S/D task began with the presentation of the upper picture (the sample). After the pigeon pecked the sample once, the lower picture (the probe) and the white rectangle were added to the display. If the sample and probe were the same, a peck to the probe was reinforced with a tone and access to the food hopper for $4-6 \mathrm{sec}$. If the pictures were different, a peck to the white rectangle was reinforced with the same tone and same amount of hopper access time. Incorrect choices were not reinforced. Each trial (correct or incorrect) was followed by a 15 -sec intertrial interval. Beginning on the second training session, incorrect choices resulted in the presentation of the correction procedure. Following the pigeon's incorrect choice, the chamber was darkened for a 15 -sec timeout. After the timeout, the incorrect trial was then repeated. If the pigeon made the correct response, the next trial was presented. If the response was incorrect, then, according to the correction procedure, the trial was repeated until a correct response was made. Session accuracy, however, was based only on the first response to 
A
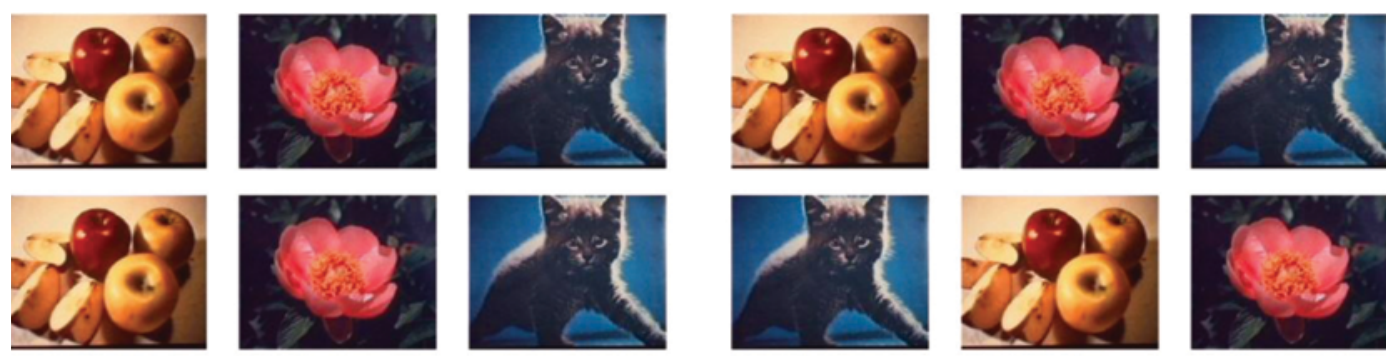

Same

Different
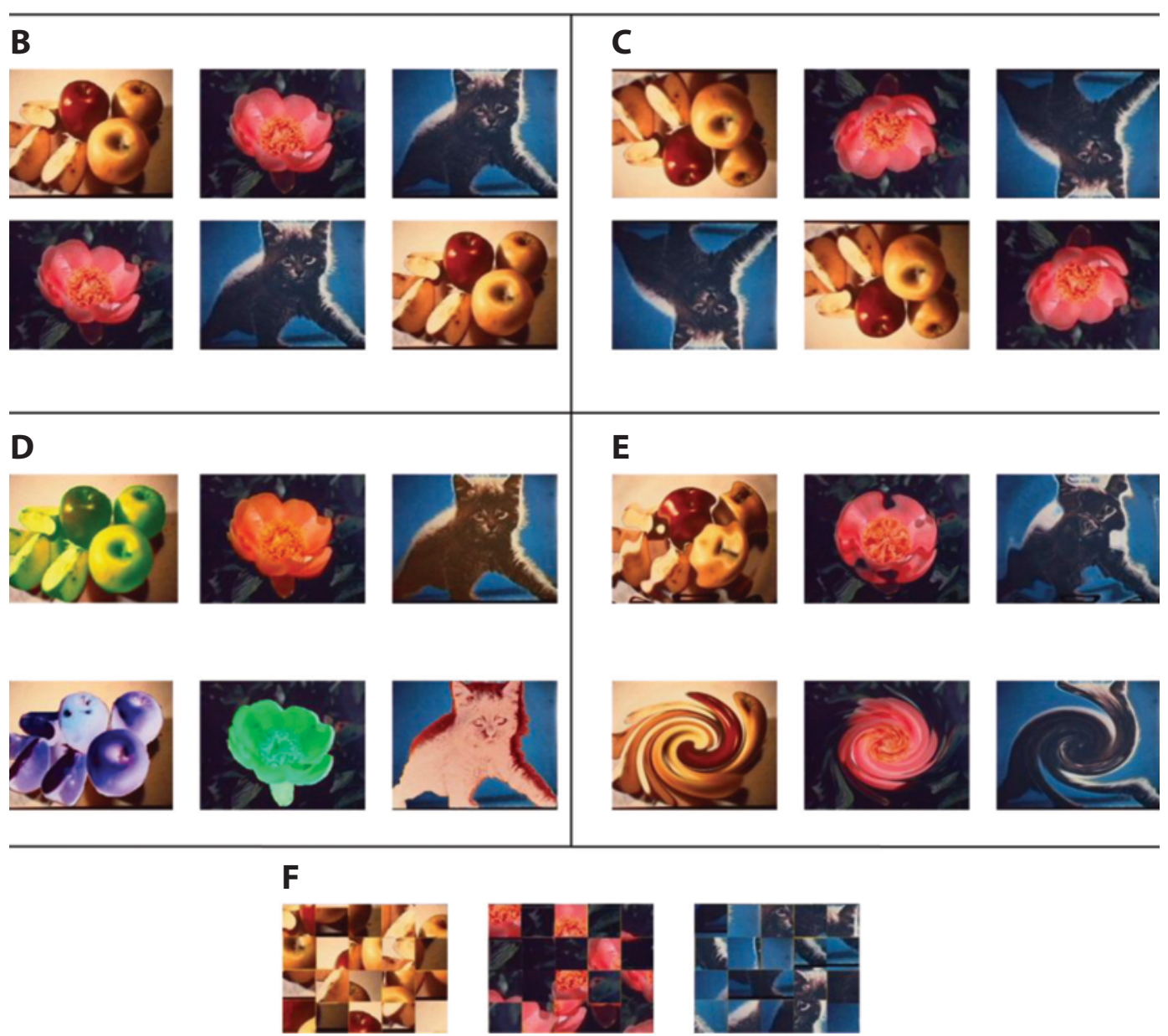

Figure 1. Training and testing stimulus displays. (A) Training displays. (B) Untrained-set testing displays. (C) Stimulus-inversion testing displays (different trials only). (D) Top row, Color Test 1; bottom row, Color Test 2.

(E) Top row, Shape Test 1; bottom row, Shape Test 2. (F) Shape Test 3.

each individual trial. Additionally, over the first 15 training sessions, the number of times that the pigeons were required to peck the upper picture was gradually increased to 20 pecks.

Each daily session contained 96 trials (48 same, 48 different). The sequence of same and different trials was pseudorandomly constructed and varied every day. Each pigeon was allotted $2 \mathrm{~h}$ to complete the task in the chamber. If a 96-trial session was not completed within these $2 \mathrm{~h}$, it was continued the next day. Training sessions continued daily until the performance level was $80 \%$ (or better) for three consecutive sessions. Then the correction procedure was removed, and training was continued until performance was again
$80 \%$ (or better) for 3 consecutive days. Once this criterion was met, the pigeons entered the testing phase of the experiment.

Untrained-set testing. As mentioned previously, initial training was conducted with only three of the six possible different trials. This training provided an opportunity to test whether the pigeons learned item-specific associations to the training displays, or whether they were using a relational rule, by testing with the three untrained different displays. If relational learning had occurred, the pigeons would be expected to transfer well to the untrained different displays, because they would be responding on the basis of the relationship between the stimuli in the pair. However, if item-specific associations were 
learned, there should be little or no transfer throughout the test. The pigeons would have learned either if-then associations or configural patterns, and neither would be expected to produce good transfer to the untrained-set pairs. The untrained-set displays appear in Figure 1B.

The untrained-set testing was conducted for eight sessions with 9 test trials inserted into each 96-trial training session, replacing 9 training different trials. Thus, each session contained 3 of each untrained-set display plus 87 baseline training trials. Throughout the untrained-set testing, and in all of the subsequent tests in this study, correct responses on test trials were reinforced, just as on baseline trials.

Novel-Stimulus Test 1. Immediately following the untrainedset testing, the pigeons were tested with novel stimuli to determine whether they had learned the S/D abstract concept. This test consisted of six sessions with 10 novel-stimulus test trials (5 same and 5 different) randomly intermixed with 86 baseline-training trials to make up each 96-trial session. These test trials contained novel, never-beforeseen stimuli. Each novel stimulus was presented on only 1 trial. Fifteen novel items were used in each daily session ( 5 for the same trials, 10 for the different trials), such that a total of 90 novel items were tested over the course of the six novel-stimulus test sessions.

\section{Results and Discussion}

\section{Initial Acquisition}

The pigeons rapidly acquired the S/D task in approximately 20 sessions $(M=1,920$ trials; range $=1,728-$ 2,304 trials). Acquisition of the task for each pigeon is shown in Figure 2. Acquisition was comparable to that of pigeons trained in a similar task with eight items and with all possible stimulus combinations in Katz and Wright (2006; $M=2,988$ trials, range $=1,900-4,500$ trials $)$. In fact, a one-way ANOVA indicated that there was no significant difference in acquisition rates between the eight-item group from Katz and Wright and the threeitem group trained in the present study $[F(1,5)=1.88$, $p=.23]$. In the present three-item task, pigeons learned 3 same trials and 3 different trials - a total of 6 stimulus displays. This learning requirement is in contrast with that of pigeons trained with eight items, which had to learn 56 possible different trials and 8 same trials, for a total of 64 stimulus displays. Group learning differences as a function of number of stimulus combinations

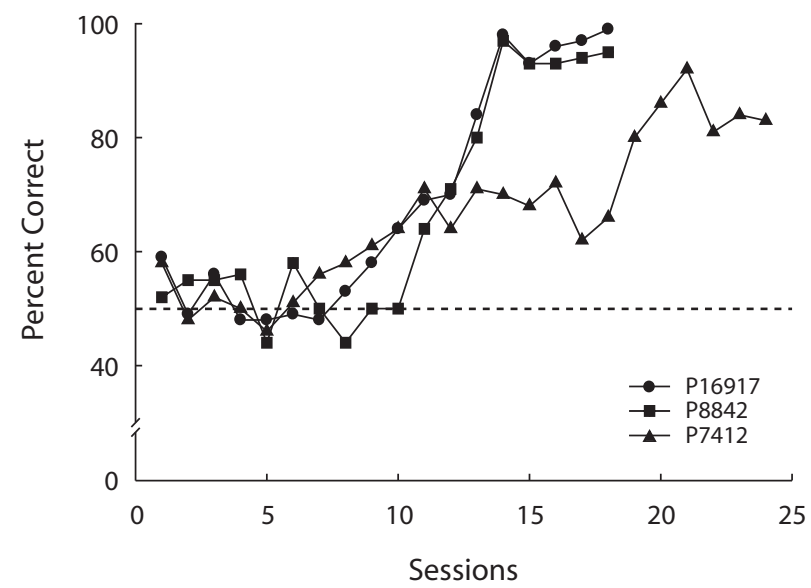

Figure 2. Percentage correct for each 96-trial session for each pigeon during acquisition. The dashed line represents chance performance.
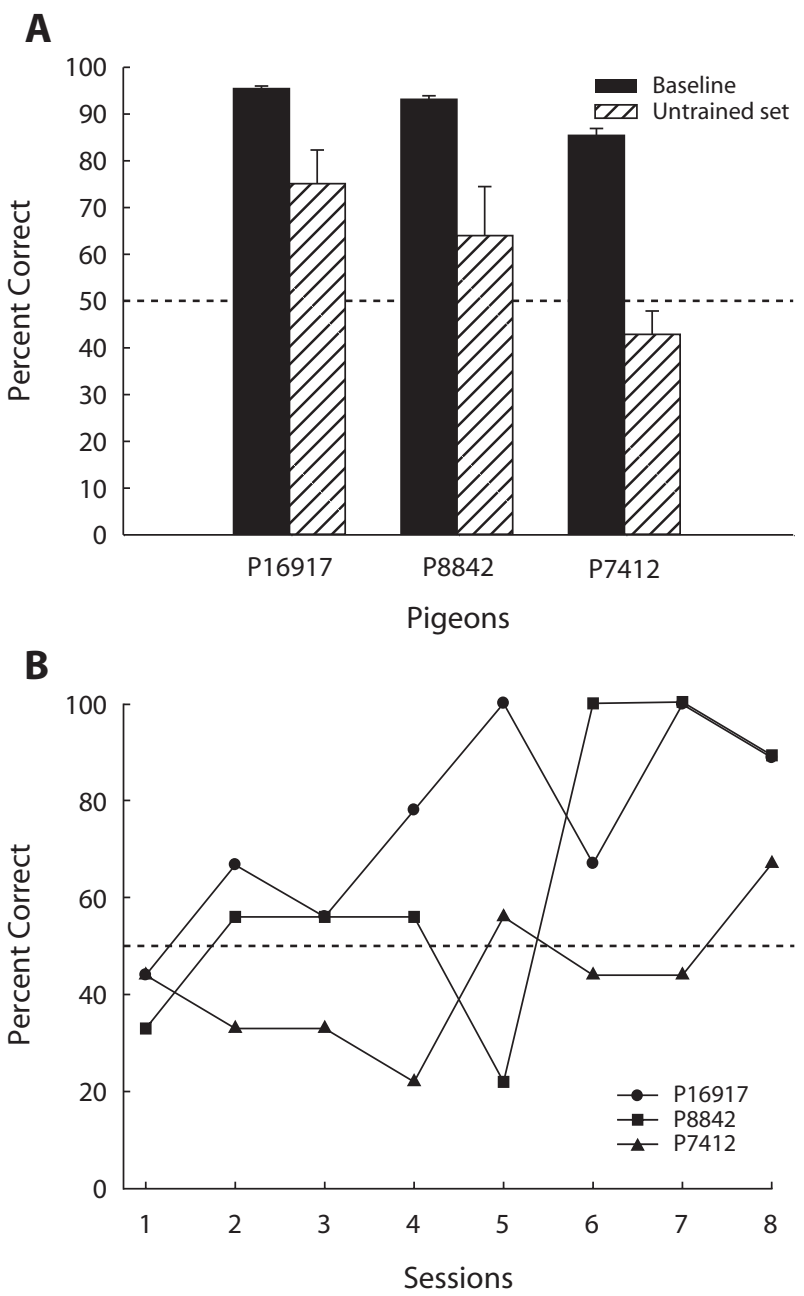

Figure 3. Untrained-set performance. (A) Mean percentages correct for baseline and untrained-set test, averaged over the eight test sessions for each pigeon. Error bars represent standard errors of the means. (B) Percentages correct for each pigeon in each of the eight untrained-set testing sessions. The dashed line represents chance performance.

would be expected if pigeons in both the three-item and eight-item tasks learned something specific to their training displays, as opposed to the abstract concept of same and different, or even a restricted-domain relational rule. Similarities in acquisition rates between the two groups suggest both that they may be using similar learning strategies and that these strategies are not entirely item specific. The most likely explanation for how pigeons trained with 6 stimulus pairs and pigeons trained with 64 stimulus pairs could have acquired at the same rate is that learning in both groups was based on the relationship of the stimuli presented in each trial. This explanation would hold even if the relational learning was confined to the training stimuli, such that accurate performance would not transfer to novel stimuli.

\section{Untrained-Set Test}

The mean results from the untrained-set test are shown in Figure 3A. Mean performances for 2 pigeons, P16917 and 


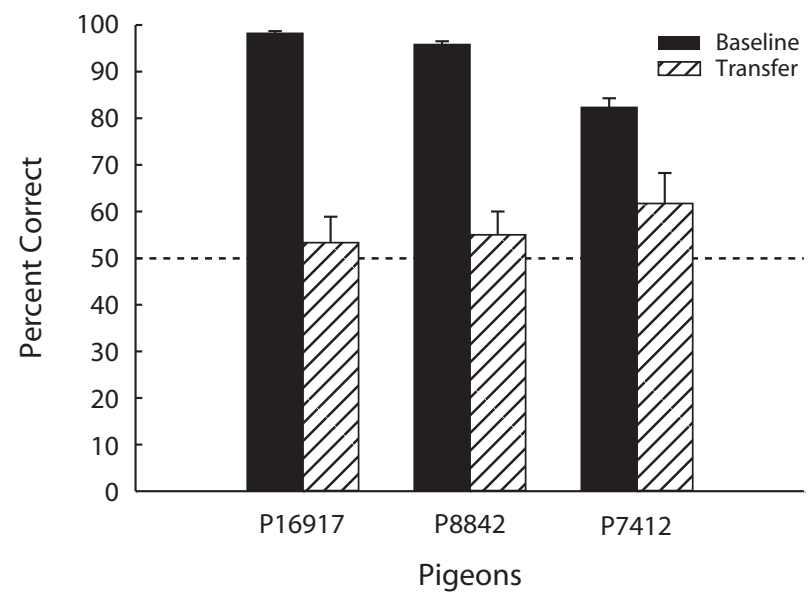

Figure 4. Novel-stimulus transfer test performance. Mean percentages correct for baseline and novel-stimulus test, averaged over the six test sessions for each pigeon. Error bars represent standard errors of the means. The dashed line represents chance performance.

P8842, were significantly greater than chance (binomial test, $p<.005$ for both pigeons). P7412's performance did not differ significantly from chance $(42.9 \%$ correct, binomial test, $p>.05$ ). It is somewhat informative to break these results down by test day to look at trends in their test performances. The daily results for the 3 pigeons are shown in Figure 3B. All of the pigeons started out below chance performance on Day 1 (P16917, 44.0\%; P8842, $33.0 \%$; and $\mathrm{P} 7412,44.0 \%$ correct). This is an important result, because it shows that the pigeons initially either did not compare the two familiar stimuli on each trial or were uncertain as to how to use this relational information and how to associate it with the correct response.

The 2 pigeons P16917 and P8842 showed a rapidly increasing percentage of correct responses to each of the untrained displays across days, confirmed by regression analyses $\left[R^{2}=.59, F(1,6)=8.51, p=.03\right.$, and $R^{2}=$ $.49, F(1,6)=5.74, p=.05$, respectively]. Considering that, after only 24 presentations of each of the untrainedset configurations, P16917's performance was as good as it was with the initial training items and P8842's performance was rapidly approaching baseline, they may have developed a relational strategy during the test. Since item-specific learning involves memorizing the correct response to each pair of items, it might require more than the 72 untrained-set trials presented during the test. P7412 represents a stronger case for item-specific learning, because this pigeon showed only a slight increase in performance over the 8 test days [regression analysis: $R^{2}=.34$, $F(1,6)=3.14, p=.13]$.

\section{Novel-Stimulus Test 1}

Performance in Novel-Stimulus Test 1 is shown in Figure 4, which presents near-chance performance by all 3 pigeons. Thus, there was little or no evidence for noveltransfer or abstract-concept learning, again indicating that learning was somehow tied to the training items. This lack of novel-transfer performance was to be expected, since neither pigeons nor monkeys trained with a larger set of eight items showed any significant novel-transfer performance (Wright \& Katz, 2006).

\section{EXPERIMENT 2}

The results of Experiment 1 suggest that learning the S/D task was initially tied to the specific training items (i.e., item-specific learning) or was in some way restricted to the domain defined by the training (stimuli). Since the pigeons had learned the task with stimuli in their normal upright orientations, turning the items upside down would invert the shape and color features in a top-down order and would alter the appearance of the individual stimuli that composed each pair. This change would be expected to disrupt - at least temporarily — any learning based on item-specific associations. It should be mentioned that there should be no novelty aversion or disruption due to novel features (e.g., novel colors) other than orientation. Any disruption in performance would have to be based on an unfamiliar appearance of either the individual items or the training pair configurations.

\section{Method}

The subjects, basic procedure, and apparatus were the same as those used in Experiment 1.

\section{Stimulus-Inversion Test}

The stimulus-inversion test began immediately following the end of the novel-transfer test in Experiment 1. Nine stimulus-inversion test trials were substituted into each of six 96-trial sessions. Each test trial consisted of an original same or different training configuration (the top and bottom positions were not changed), but both the sample and the probe were turned upside down (Figure 1C). During each test session, there were either 4 same and 5 different, or 5 same and 4 different trials. The total numbers of same and different test trials were balanced over the six daily sessions such that each pigeon was tested with a total of 27 same and 27 different test trials.

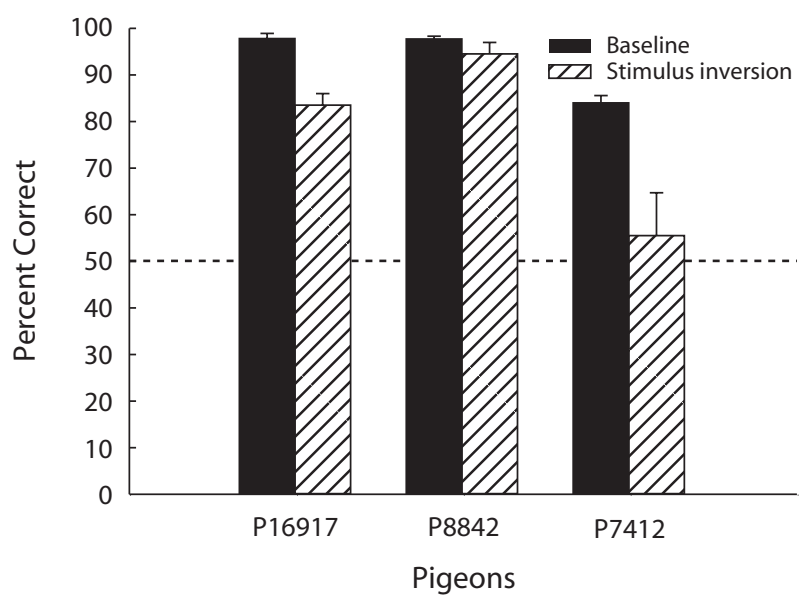

Figure 5. Stimulus-inversion test performance. Mean percentages correct for baseline and Stimulus-Inversion Test 1, averaged over the six test sessions for each pigeon. Error bars represent standard errors of the means. The dashed line represents chance performance. 


\section{Results and Discussion}

Figure 5 shows that pigeons P16917 and P8842 both performed well in the stimulus-inversion test. Their performances were well above chance $(83.5 \%$ and $94.5 \%$ correct, respectively). P16917's performance-although significantly different from baseline [one-way ANOVA, $F(1,10)=28.5, p<.001]$ — was significantly greater than chance (binomial test, $p<.005$ ). P8842's performance was not significantly different from baseline [one-way ANOVA, $F(1,10)=1.56, p=.24]$. Additionally, rapid learning cannot account for P16917's and P8842's high performance in this test, since these subjects were both well above chance on the first day of testing (78\% and $100 \%$, respectively).

Pigeon P7412 did not perform well in the stimulusinversion test; this subject's performance (55.5\% correct) was not significantly better than chance (binomial test, $p>.05)$. This result provides further evidence that P7412 had learned the task item-specifically.

The results of the stimulus-inversion test suggest that P16917 and P8842 may have developed a more general rule with which to perform the task, unlike the results of P7412, which were suggestive of an item-specific strategy. Given that P16917 and P8842 failed Novel-Transfer Test 1, we know that they had not learned the abstract concepts of same and different. Together, these results suggest that their processing was relational but restricted domain. The restricted domain is broader than just the training stimuli themselves, because it includes the upside-down images presented in the stimulus-inversion tests.

How restricted or broad is the domain? In our next experiment, we attempted to explore two dimensions that ought to contribute to the breadth of this visual domainshape and color.

\section{EXPERIMENT 3}

Color and shape (form) are two of the defining features of all color picture stimuli. We manipulated these features to explore how they might affect the apparent restricteddomain relational learning of P16917 and P8842, as well as the apparent item-specific learning of P7412. We tested two degrees of color and shape changes in order to provide two points on the continuum of the restricted domain and to test how the changes would affect P7412's apparent item-specific learning. We then retested novel-item transfer. This second novel-item transfer test was conducted to rule out the possibility that, following the extensive training and testing that occurred throughout Experiments 2 and 3, the experience with the testing stimuli and the further experience in the task itself could have facilitated some abstract-concept learning.

\section{Method}

The subjects, basic procedure, and apparatus were the same as those used in Experiments 1 and 2.

\section{Testing Procedure}

Shape and Color Tests 1. The first phase of shape and color testing began immediately following Experiment 2 . These tests were conducted for 8 consecutive testing days. Nine test trials were substituted for 9 training trials in each 96-trial daily session, and colorchange trials and shape-change trials alternated daily. For example, if a pigeon was tested with color-change trials on Day 1, this pigeon was tested with shape-change trials on Day 2 , and so on. Since there were 9 test trials per session, on one day, there were 4 same and 5 different test trials, but, on the next day, there were 5 same and 4 different trials, such that by the end of the test, 18 same and 18 different trials would have been run for both color and shape. Color-change trials were created by taking the three original stimuli and slightly changing the color of the item in the foreground but not that of the background (see Figure 1D, top row). For instance, the dark gray cat was changed to brown, but the blue background remained the same. The pink flower was changed to orange, but the background stayed dark green. Likewise, the yellow and red apples were changed to yellow green, but the background stayed yellow/tan. Shape-change trials were created by using the ripple feature in Jasc Paint Shop Pro Version 7.04 on each item (Figure 1E, top row). Original training set configurations were the pairings used in all test trials.

Shape and Color Tests 2. The second phase of shape and color testing began immediately following the first phase. The numbers of test days and trials remained the same as in Phase 1, but, in this phase, the color and shape changes were more pronounced. Colorchange trials were created by drastically changing the color of the item, while keeping the color of the background the same (see Figure 1D, bottom row). For instance, the pink flower was changed to bright lime green, but the background remained dark green. The dark gray cat was changed to pink and white, and the background remained blue; and the yellow and red apples were changed to purple and blue, but the background remained yellow/tan. Shape-change trials were created by using the twirl feature in Jasc Paint Shop Pro, which made pronounced changes to the shapes of the stimuli (Figure $1 \mathrm{E}$, bottom row).

Shape Test 3. Following Shape and Color Tests 2, a third shape test was conducted. This test lasted 4 days, with 9 test trials in each 96-trial daily session (4 same and 5 different or 5 same and 4 different trails, for a total of 18 same and 18 different). This test also involved a pronounced change in shape. Each training stimulus was divided into 20 squares, and the squares were randomly repositioned to create a scrambled image (Figure 1F). The scrambling of the images was accomplished using Jasc Paint Shop Pro. In the two prior shape tests, the basic positioning of stimuli was the same, but the centers of the images were warped. In this test, basic positional cues were disrupted to see whether this (arguably more drastic) change in shape would disrupt the pigeons' performance.

Novel-Stimulus Test 2. After Shape Test 3, pigeons were tested (for a second time) with completely novel stimuli. These did not include any of the items tested in Novel-Stimulus Test 1 . This test lasted 6 days, with 10 test trials (5 same and 5 different) in each 96trial session. Fifteen completely novel items were used on each test day (5 for the same trials and 10 for the different trials).

\section{Results}

\section{Shape and Color Tests 1}

As is shown in Figure 6A, P16917, P8842, and P7412 all performed significantly better than chance in Shape Test 1 (binomial test, $p<.005$ for all 3 pigeons). Also, a one-way ANOVA revealed that none of the pigeons' test performance was significantly different from their baseline performance $[\mathrm{P} 16917, F(1,6)=0.01, p=.93$; $\mathrm{P} 8842, F(1,6)=0.59, p=.47 ; \mathrm{P} 7412, F(1,6)=0.86, p=$ .39]. Rapid acquisition cannot account for P16917's and P8842's high performance, because Day 1 performances were $100 \%$ for both pigeons. P7412 improved slightly; this subject's Day 1 test performance was 56\%. However, the improvement was small enough that regression analy- 
A

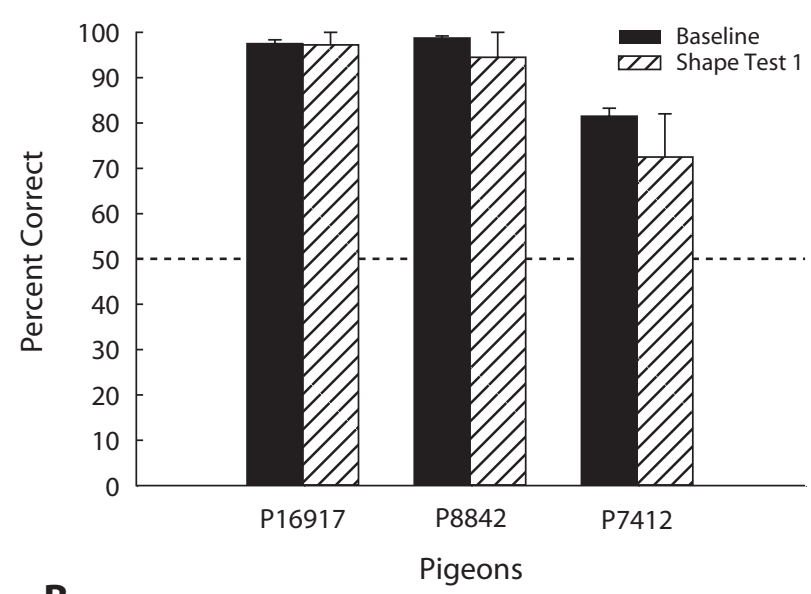

B

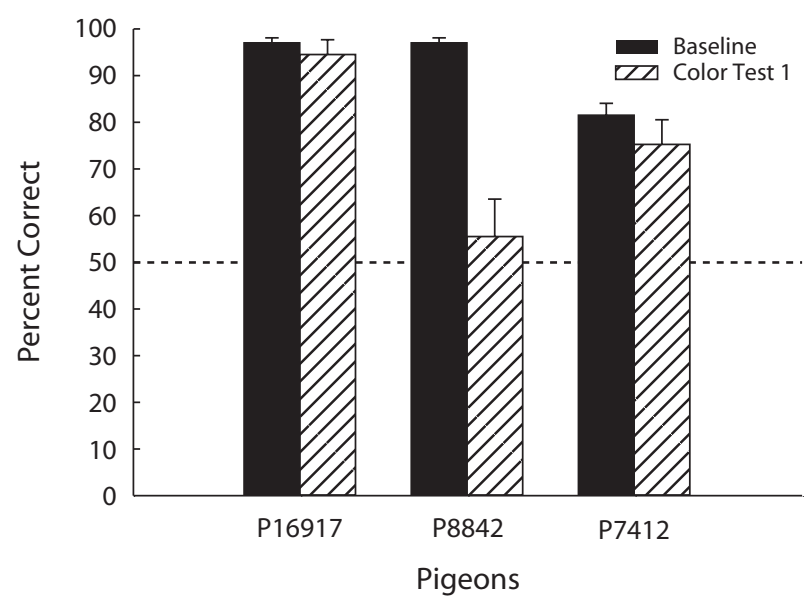

Figure 6. Shape and Color Tests 1 performance. (A) Mean percentages correct for baseline and Shape Test 1 , averaged over the four test sessions for each pigeon. (B) Mean percentages correct for baseline and Color Test 1, averaged over the four test sessions. Error bars represent standard errors of the means. The dashed line represents chance performance.

ses did not reveal a significant increase in performance across sessions $\left[R^{2}=.2, F(1,2)=0.5, p=.55\right]$.

As is shown in Figure 6B, P16917's and P7412's performances were significantly better than chance in Color Test 1 (binomial test, $p<.005$ for both pigeons) and were not significantly different from their baseline performances [one-way ANOVA, $F(1,6)=0.56, p=.48$, and $F(1,6)=$ $1.14, p=.32$, respectively]. P16917's high performance cannot be accounted for by rapid learning, because this subject's Day 1 performance was $100 \%$. However, regression analyses revealed that P7412 improved quickly across sessions; this subject's Day 1 performance was only $67 \%\left[R^{2}=.89, F(1,2)=16.33, p=.05\right]$. P8842's transfer performance was not significantly different from chance on Color Test 1 (binomial test, $p>.05$ ).

\section{Shape and Color Tests 2}

Results from Shape Test 2 are shown in Figure 7A. All 3 pigeons again performed significantly better than chance, as was shown by a binomial test ( $\mathrm{P} 16917, p<.005 ; \mathrm{P} 8842$, $p<.005$; P7412, $p<.01)$. P16917's and P8842's performances $(97.25 \%$ and $91.75 \%$ correct, respectively) did not differ significantly from baseline [one-way ANOVA, $F(1,6)=0.40, p=.55$, and $F(1,6)=0.82, p=.40$, respectively]. Additionally, these subjects maintained high transfer performance throughout the test, beginning with scores of $100 \%$ and $78 \%$ correct, respectively, on Day 1 of testing. Although P7412's transfer performance was significantly above chance, this subject's test performance was significantly different from baseline, at $69.75 \%$ correct [one-way ANOVA, $F(1,6)=14.04, p=.01$ ]. In fact, this subject's Day 1 test performance was $67 \%$, indicating that he did not improve much throughout the test.

Results from Color Test 2 are shown in Figure 7B. Overall, transfer performance was not significantly different from chance for all 3 pigeons, as was confirmed by a binomial test ( $p>.05$ for all pigeons).
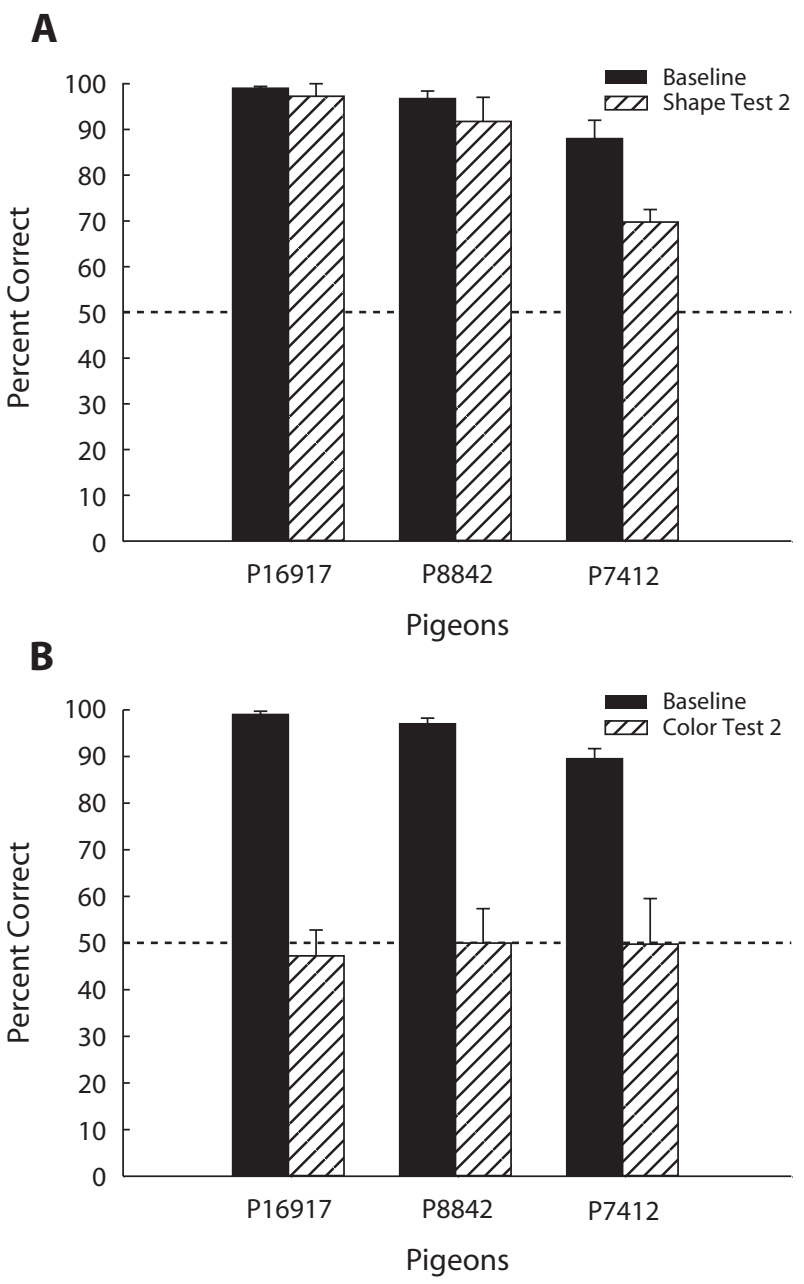

Figure 7. Shape and Color Tests 2 performance. (A) Mean percentages correct for baseline and Shape Test 2 , averaged over the four test sessions for each pigeon. (B) Mean percentages correct for baseline and Color Test 2, averaged over the four test sessions for each pigeon. Error bars represent standard errors of the means. The dashed line represents chance performance. 


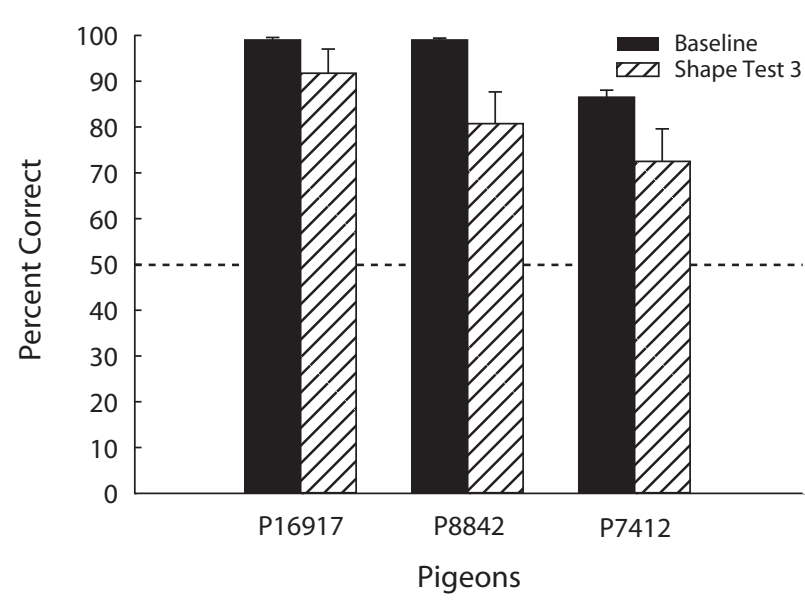

Figure 8. Shape Test 3 performance. Mean percentages correct for baseline and Shape Test 3 , averaged over the four test sessions for each pigeon. Error bars represent standard errors of the means. The dashed line represents chance performance.

\section{Shape Test 3}

The results of Shape Test 3 are shown in Figure 8. All 3 pigeons performed significantly better than chance, as was confirmed by a binomial test $(p<0.005$ for all 3 pigeons). In fact, P16917's and P7412's transfer performances were not significantly different from their baseline performances [one-way ANOVA, $F(1,6)=1.87$, $p=.22$, and $F(1,6)=3.71, p=.10$, respectively]. Although P8842's performance did differ significantly from this subject's baseline performance [one-way ANOVA, $F(1,6)=6.93, p=.04]$, all 3 pigeons nonetheless performed well on this test, suggesting that even this drastic shape change did not substantially disrupt transfer performance. Additionally, regression analyses revealed that rapid learning could not account for high performance in the task. P16917's, P8842's, and P7412's Day 1 test performances were greater than chance $(78 \%, 67 \%$, and $67 \%$, respectively), and regressions were not significant $\left[R^{2}=\right.$ $.16, F(1,2)=0.39, p=.60 ; R^{2}=.01, F(1,2)=0.02, p=$ .90 ; and $R^{2}=.04, F(1,2)=0.08, p=.8$, respectively].

\section{Novel-Stimulus Test 2}

Overall, the pigeons' performances in Novel-Stimulus Test 2 were not significantly different from their performances in Novel-Stimulus Test 1 [two-way repeated measures ANOVA with pigeon (P16917, P8842, P7412) and test (Test 1, Test 2) as factors, $F(2,2)=0.86, p=.43$ ], since these subjects performed at $67 \%, 60 \%$, and $60 \%$ correct, respectively.

\section{Discussion}

The fact that P16917 and P8842 performed well with both subtle (Shape Test 1) and drastic (Shape Tests 2 and 3) changes in shape provides converging evidence supporting the conclusions drawn from the stimulus-inversion tests. Their high performance in the stimulus-inversion test and all three shape tests supports the notion of a restricteddomain relational strategy.
Although P16917 performed well in Color Test 1, P8842 did not. The changes in Color Test 1 seemed slight but were apparently drastic enough to disrupt the performance of P8842. Additionally, the degree of color change presented in Color Test 2 was drastic enough to disrupt the performance of all 3 pigeons, since their performance fell to chance. The drastic color changes essentially transformed the training items into the equivalent of novel items. Color appears to be a more important feature for these subjects than shape is.

Interestingly, P7412 performed well in Color Test 1 (but not in Color Test 2) and in all three shape tests. As for P16917 and P8842, it seems that color is more important than shape for this subject. However, recall that P7412 performed poorly in the stimulus-inversion test, which essentially involved a change in shape. This discrepancy in performance between the stimulus-inversion test and the shape tests is difficult to explain, but perhaps the experience that this subject gained with transformed items during the stimulus-inversion test facilitated higher performance in the shape tests.

Finally, the poor performance by all 3 pigeons in NovelStimulus Test 2 confirms that, even after extensive training and testing during Experiments 2 and 3 , their performance with novel stimuli did not change. This is important because it shows that the relational processing by P16917 and P8842 remained virtually unchanged across the experiments in terms of its being restricted domain. This result is also consistent with the conclusion that P7412 was using an item-specific strategy, since this subject failed to transfer his performance to novel stimuli.

These results bring us back to the question of how to define the domain of items to which the pigeons are able to apply their relational concept. It seems likely that the domain is fairly broad, to the degree that a range of shape changes can be tolerated. However, our results indicate that the range is less broad for color.

\section{GENERAL DISCUSSION}

The results of the untrained-set test in Experiment 1 suggest that 2 pigeons (P16917 and P8842) likely adopted a relational strategy, since their performances were significantly better than chance, whereas the third pigeon (P7412) may have employed an item-specific strategy, since his performance was at chance. These results were further confirmed by the stimulus-inversion test in Experiment 2, in which P16917 and P8842 performed significantly above chance, thereby reinforcing the suggestion that they were engaged in relational learning. Furthermore, P7412 performed poorly in the stimulus-inversion test, which is what would be expected if this subject were using an item-specific strategy. Nevertheless, the results of the novel-transfer test in Experiment 1 indicated that all 3 pigeons learned something that was specific to the training stimuli. This lack of novel-item transfer is what would be expected of P7412 on the basis of evidence from other tests suggesting item-specific learning. The lack of novel-stimulus transfer by P16917 and P8842 suggests that these 2 pigeons had learned a restricted-domain re- 
lational strategy. In order to begin exploring controlling features for item-specific and restricted-domain relational learning, color and shape were manipulated. The results indicate that color was a more critical feature than was shape for all 3 pigeons, suggesting that, for P16917 and P8842, the domain of relational learning might be broader in the shape dimension than in the color dimension in this S/D task.

Researchers have previously considered the relative importance of shape and color to pigeons in other types of cognitive tasks, such as stimulus-discrimination and stimulus-grouping tasks. Reynolds (1961) found that pigeons tested in a go/no-go discrimination task divided with respect to whether they were under control of a white triangle (shape) or the red background (color). By contrast, all of the pigeons in the present study seemed to treat color as the more critical feature. In line with our results, Kirsch, Kabanova, and Güntürkün (2008) demonstrated in an MTS task that color was primarily the controlling feature, not shape. Cook, Katz, and Cavoto (1997) also found that color was the controlling feature in a same/ different task involving an oddity-discrimination task. Additionally, Lazareva, Vecera, Levin, and Wasserman (2005) found that color rather than shape was the critical cue in a one-object versus two-object discrimination task. It appears that color may be the overriding perceptual feature when compared with shape in many visual cognitive tasks with pigeons, and color may therefore define to a large extent the nature of what is restricting the domain for the relational learning that occurred for pigeons P16917 and P8842 in the present study. As in the results from the Reynolds study, individual differences arose in the present study, with test results suggesting that P7412 learned the task item-specifically.

Previous researchers have typically concluded that, when animals acquire the same/different task (particularly with a small training set size) but initially fail to learn the full abstract concept, they must be using some type of item-specific strategy, such as configural or if-then rule learning (e.g., Carter \& Werner, 1978; Premack, 1978). As a result, the fact that 2 of the 3 pigeons appeared to learn relationally with only six trial types - albeit in a restricteddomain manner - was highly unexpected, because the experiment was designed to facilitate item-specific strategies. However, relational learning with a small set of stimuli is not unheard of; Oden et al.'s (1988) infant chimpanzees did demonstrate full abstract-concept learning in the MTS task after training with only two stimuli.

Although the present experiments may be among the first showing that pigeons trained with a small set size learned the task relationally (i.e., restricted-domain relational learning), such a result may actually have occurred in other experiments but gone unnoticed. For instance, the pigeons in Katz and Wright (2006) that were trained with eight items undoubtedly failed to transfer to novel items, but that result does not preclude the possibility that the eight-item pigeons were also using a restricteddomain relational strategy. Those pigeons were never tested with the untrained-set or stimulus-inversion tests, which were shown here to be useful tests in identifying the learning strategies that each of the pigeons was likely to be using.

The notion of restricted-domain relational learning may also help explain the development of full-concept learning following set-size expansion seen in Katz and Wright (2006). As set size increased, the domain in which the pigeons were able to apply their relational learning became larger as well. With each subsequent set-size expansion, the domain encompassed a larger variety of stimuli, and novel-stimulus transfer improved. Once the set size became sufficiently large (e.g., 256 items), full transfer occurred, because the domain covered a greater range of stimuli. The set-size transfer functions were continuous, suggesting that the domain expanded. There was no abrupt step in the transfer function, which might be expected with a learning strategy shift from item-specific learning to relational learning.

The transition from restricted-domain relational learning to full application of the abstract concept likely proceeds in stages. In the first stage, the subjects learn to compare the relationship between the sample and the probe in order to make a judgment of same or different, but only in the cases in which the sample and probe items fall within the restricted domain. Later, in the second stage, as the pigeons' set size is expanded and the domain is broadened, they learn to use relational processing to solve the task with a much broader range of items, including other colors and color combinations. There is a parallel phenomenon seen in the development of abstract thinking and analogical reasoning in children. Doumas, Hummel, and Sandhofer (2008) explained, "Children go through a domain-specific relational shift in which they transition from representing a domain in terms of its characteristic features to representing it in terms of its characteristic relations as well"' (p. 21). Although Doumas et al. are referring to the development of a more advanced cognitive process than simple same/different judgments, the framework with which they describe the development is relevant to our data as well. Pigeons may begin by perceiving the same and different aspects of the training pairs and associating these percepts with particular responses that are reinforced. They may then transition to representing these relationships more generally and applying it to new exemplars, once they fully understand the abstract relation of sameldifferent.

The question remains, why would the pigeon not just learn the general rule of same and different in the first place and apply it to all stimuli? One potential answer is that applying the general rule to stimuli that lie beyond the restricted domain requires a degree of cognitive flexibility. As Watanabe (2006) suggested, "a flexible systems costs more than a fixed system" (p. 632). In a task with a small set size, the benefits of operating outside of the domain are few; thus, the pigeon does not need to be cognitively flexible. However, as set size increases, the cost of a fixed system is high, since flexible behavior is necessary to successfully solve the task. Thus, with increasingly large set sizes, the costs of being cognitively flexible decrease and the costs of operating with a fixed system increase, until the flexible system ultimately wins out and full transfer is achieved. 


\section{AUTHOR NOTE}

This research was supported by NIH Grants 5 T32 NS07467, MH061798, and MH-072616, and by NSF Grant IBN-0316113. Correspondence and reprint requests may be addressed to any of the authors: L. C. Elmore, A. A. Wright, or J. J. Rivera, Department of Neurobiology and Anatomy, University of Texas Medical School at Houston, P.O. Box 20708, Houston, TX 77225; J. S. Katz, Department of Psychology, 226 Thach Hall, Auburn University, Auburn, AL 36849 (e-mail: anthony.a.wright@uth.tmc.edu, lauren.c.elmore@uth.tmc.edu, jacquelyne.j.rivera@uth.tmc.edu, or katzjef@auburn.edu).

\section{REFERENCES}

Bhatt, R. S., \& Wright, A. A. (1992). Concept learning by monkeys with video picture images and a touch screen. Journal of the Experimental Analysis of Behavior, 57, 219-225.

Bovet, D., \& Vauclair, J. (2001). Judgment of conceptual identity in monkeys. Psychonomic Bulletin \& Review, 8, 470-475.

CARTER, D. E., \& Werner, T. J. (1978). Complex learning and information processing by pigeons: A critical analysis. Journal of the Experimental Analysis of Behavior, 29, 565-601.

Cook, R. G., Cavoto, K. K., \& Cavoto, B. R. (1995). Same-different texture discrimination and concept learning by pigeons. Journal of Experimental Psychology: Animal Behavior Processes, 21, 253-260.

Cook, R. G., Katz, J. S., \& Cavoto, B. R. (1997). Pigeon samedifferent concept learning with multiple stimulus classes. Journal of Experimental Psychology: Animal Behavior Processes, 23, 417-433.

Doumas, L. A. A., Hummel, J. E., \& Sandhofer, C. M. (2008). A theory of the discovery and predication of relational concepts. Psychological Review, 115, 1-43.

JAMES, W. (1950). The principles of psychology (Vol. 1). New York: Dover. (Original work published 1890)

KatZ, J. S., \& Wright, A. A. (2006). Same/different abstract-concept learning by pigeons. Journal of Experimental Psychology: Animal Behavior Processes, 32, 80-86.

Katz, J. S., Wright, A. A., \& Bachevalier, J. (2002). Mechanisms of sameldifferent abstract-concept learning by rhesus monkeys (Macaca mulatta). Journal of Experimental Psychology: Animal Behavior Processes, 28, 358-368.

Kirsch, J. A., Kabanova, A., \& GÜNTÜrKüN, O. (2008). Grouping of artificial objects in pigeons: An inquiry into the cognitive architecture of an avian mind. Brain Research Bulletin, 75, 485-490.

LASHLEY, K. S. (1930). The mechanism of vision: I. A method for rapid analysis of pattern vision in the rat. Journal of Genetic Psychology, 37, 453-460.

Lazareva, O. F., Vecera, S. P., Levin, J., \& Wasserman, E. A. (2005). Object discrimination by pigeons: Effects of object color and shape. Behavioural Processes, 69, 17-31.
Oden, D. L., Thompson, R. K. R., \& Premack, D. (1988). Spontaneous transfer of matching by infant chimpanzees (Pan troglodytes). Journal of Experimental Psychology: Animal Behavior Processes, 14, 140-145.

PePperberG, I. M. (1987). Acquisition of the same/different concept by an African Grey parrot (Psittacus erithacus): Learning with respect to categories of color, shape, and material. Animal Learning \& Behavior, 15, 423-432.

Premack, D. (1978). On the abstractness of human concepts: Why it would be difficult to talk to a pigeon. In S. H. Hulse, H. Fowler, \& W. K. Honig (Eds.), Cognitive processes in animal behavior (pp. 423451). Hillsdale, NJ: Erlbaum.

Premack, D. (1983a). Animal cognition. Annual Review of Psychology, 34, 351-362.

Premack, D. (1983b). The codes of man and beasts. Behavioral \& Brain Sciences, 6, 125-167.

Premack, D., \& Premack, A. J. (1983). The mind of an ape. New York: Norton.

Reynolds, G. S. (1961). Attention in the pigeon. Journal of the Experimental Analysis of Behavior, 4, 203-208.

Wasserman, E. A., Hugart, J. A., \& Kirkpatrick-Steger, K. (1995). Pigeons show same-different conceptualization after training with complex visual stimuli. Journal of Experimental Psychology: Animal Behavior Processes, 21, 248-252.

Watanabe, S. (2006). The neural basis of cognitive flexibility in birds. In E. A. Wasserman \& T. R. Zentall (Eds.), Comparative cognition: Experimental explorations of animal intelligence (pp. 619-636). Oxford: Oxford University Press.

Wright, A. A. (1997). Concept learning and learning strategies. Psychological Science, 8, 119-123.

Wright, A. A., \& Katz, J. S. (2006). Mechanisms of same/different concept learning in primates and avians. Behavioural Processes, 72, 234-254.

Wright, A. A., Rivera, J. J., Katz, J. S., \& Bachevalier, J. (2003). Abstract-concept learning and list-memory processing by capuchin and rhesus monkeys. Journal of Experimental Psychology: Animal Behavior Processes, 29, 184-198.

Wright, A. A., Santiago, H. C., \& Sands, S. F. (1984). Monkey memory: Same/different concept learning, serial probe acquisition, and probe delay effects. Journal of Experimental Psychology: Animal Behavior Processes, 10, 513-529.

Wright, A. A., Shyan, M. R., \& Jitsumori, M. (1990). Auditory same/ different concept learning by monkeys. Animal Learning \& Behavior, 18, 287-294.

Zentall, T. R., \& Hogan, D. E. (1974). Abstract concept learning in the pigeon. Journal of Experimental Psychology, 102, 393-398.

(Manuscript received October 22, 2008; accepted for publication November 2, 2008.) 